\title{
EFEITO DE FITORREGULADORES SOBRE A INCIDÊNCIA DE PODRIDÃO CARPELAR EM MAÇÃS "FUJI"1
}

\author{
AIKE ANNELIESE KRETZSCHMAR ${ }^{2}$, GILMAR ARDUINO BETTIO MARODIN ${ }^{3}$, VALMIR DUARTE ${ }^{3}$, \\ ROSA MARIA VALDEBENITO-SANHUEZA ${ }^{4}$, DENIS SALVATI GUERRA ${ }^{5}$
}

RESUMO - Objetivou-se neste trabalho avaliar o efeito dos fitorreguladores CPPU (N-2-cloro-4-piridil -N-feniluréia), Promalina $\left(\mathrm{GA}_{4+7}\right.$ + BA), Benziladenina (BA) e Thidiazuron, aplicados no período entre a plena floração e a queda a de pétalas em macieiras cv. Fuji, sobre a incidência de podridão carpelar, na safra de 2001-2002 e 2002-2003. A área experimental constituiu-se de macieiras da cv Fuji/EM-9, plantio 1994 em Vacaria-RS. Foram avaliadas características de formato dos frutos e a incidência de sinais e sintomas da podridão carpelar. O Thidiazuron e a Promalina aumentaram a presença de sinais de fungos nas sementes no ciclo de 2001-2002. Houve influência de tratamentos com fitorreguladores sobre a expressão dos sintomas da podridão carpelar tanto nas maçãs caídas das plantas como naquelas avaliadas após três meses de frigoconservação nos dois ciclos vegetativos avaliados.

Termos para indexação: macieira, podridão carpelar, promalina, benziladenina, thidiazuron;

\section{EFFECTS OF GROWTH REGULATORS ON THE INCIDENCE OF MOLDY CORE IN “FUJI” APPLES}

ABSTRACT-The objective of the present study was to evaluate the effects of CPPU (N-2-cloro-4-piridil)-N-phenylurea), Promalin $\left(\mathrm{GA}_{4+7}+\mathrm{BA}\right)$, Benzyladenine (BA) and Thidiazuron, sprayed on "Fuji" apple trees at full bloom stage, on the incidence of moldy core, in the 2001/2002 and 2002/2003 seasons. The experimental area was a Fuji/EM-9 apple orchard, established in 1994 in Vacaria / RS. The apples were evaluated for fruit shape and moldy core incidence. During 2001, the seeds of carpel of apples treated with Thidiazuron and Promalin showed more fungi colonization than the control fruit. There were significant differences between treatments on moldy core rot incidence as well as in preharvest dropped apples and in apples evaluated after three months of cold storage, during the two evaluation seasons.

Index Terms: apples, moldy core, Promalin, Benzyladenine, Thidiazuron.

\section{INTRODUÇÃO}

A podridão carpelar das maçãs é uma doença que vem se tornando importante no Brasil, incidindo em todos os pomares da região produtora dessa fruta. Registros de perdas de frutos na pós-colheita mostram valores de até $15 \%$ (Czermainski et al., 2002). Kretzschmar et al., (2004) observaram que a incidência de podridão carpelar, na fase inicial de desenvolvimento, chegou a $40 \%$ em maçãs "Fuji" recém-colhidas, e aumentou para $60 \%$ após 10 dias em temperatura ambiente.

Não há, no Brasil, recomendação de controle químico eficiente para essa doença. Trabalhos realizados em outros países mostram que o controle químico foi eficiente quando o principal agente causal era o fungo Alternaria alternata (Reuveni et al., 2002; Reuveni et al., 2003).

No Brasil, essa doença ocorre principalmente na cultivar Fuji, a qual apresenta floração desuniforme e espaçada, frutos achatados e cavidade pistilar aberta, condições que facilitam a entrada de fungos que causam a podridão carpelar (Petri, 2002).

Maior incidência da podridão carpelar foi observada em maçãs que tiveram problemas durante a fertilização e a formação dos frutos e na formação de sementes causados por períodos frios, geadas tardias e chuvas durante a floração (Ellis, 1983; Boneti $\&$ Katsurayama, 1999).

Spotts (1990) relata que a doença é menos comum em frutos com uma razão comprimento/diâmetro (C/D) alta. Maçãs cv. Golden Delicious com essas características foram obtidas por Curry \& Greene (1993) com aplicação de CPPU na plena floração, e duas semanas após, os autores observaram que houve aumento da relação C/D e do peso dos frutos. Para Bangerth \& Schroder (1994), o aumento do tamanho dos frutos e a relação C/D foram proporcionais à concentração de giberelinas e citocininas aplicadas.

O efeito de CPPU e Promalina aplicados na plena floração em macieiras "Gala" e 'Fuji", em Caçador-SC, foi testado por

'(Trabalho 143-06). Recebido em: 15-09-2006. Aceito para publicação em: 18-10-2007. Parte da tese do primeiro autor, apresentada à UFRGS, para obtenção do título de Doutor.

${ }^{2}$ Eng. Agr. Dr., Prof. Faculdade de Agronomia, Centro de Ciências Agroveterinárias CAV/UDESC. E-mail: a2aak@cav.udesc.br

${ }^{3}$ Eng. Agr. Dr, Prof. Faculdade de Agronomia, UFRGS. E-mail: marodin@ufrgs.br

E-mail: valmir@ufrgs.br

${ }^{4}$ Eng. Agr. Dra. Pesquisadora Embrapa Uva e Vinho. E-mail: almazan@italnet.com.br

${ }^{5}$ Eng. Agr. MSc., Doutorando UFRGS. E-mail: dsguerra@ibest.com 
Argenta et al. (1993), que observaram que $10 \mathrm{mg} \mathrm{L}^{-1}$ de CPPU, aplicados na plena floração aumentaram o peso de maçãs "Fuji". A relação entre o uso de reguladores vegetais e a podridão carpelar foi estudada no Brasil por Amarante et al. (2003). Os autores concluíram que o Thidiazuron aplicado na plena floração aumentou a podridão calicinal ("calyx end rot") e a percentagem de frutos assimétricos, e reduziu a percentagem de cor vermelha na casca.

A partir dessas informações, o presente trabalho teve como objetivo avaliar, durante dois ciclos, o efeito de quatro fitorreguladores aplicados durante a floração sobre o formato do fruto e sobre a incidência de sinais e sintomas da podridão carpelar em maçãs "Fuji".

\section{MATERIAL E MÉTODOS}

O experimento foi instalado nos ciclos vegetativos de 20012002 e 2002-2003, na empresa Randon Agrossilvopastoril (Rasip), em um pomar de macieiras da cv. Fuji/EM-9, conduzido sob sistema de produção integrada de maçãs (PIM), tendo como polinizadora a cv. Gala. O pomar foi implantado em 1994, em solo Vacaria, latossolo bruno húmico, altitude de 950 a 1.000 metros, latitude $29^{\circ} 32^{\prime \prime}$ sul e longitude $50^{\circ} 54^{\prime} \mathrm{WGr}$, com espaçamento de 4,0 x 1,0 $\mathrm{m}$ (2.500 plantas/ha). As temperaturas médias máxima de verão e mínima de outono dessa região são $22^{\circ} \mathrm{Ce} 10^{\circ} \mathrm{C}$, respectivamente. Os dados meteorológicos durante a execução do trabalho foram coletados na Estação Experimental de Vacaria -EMBRAPA Uva e Vinho, Vacaria-RS.

Os tratamentos aplicados foram: testemunha - aplicação de apenas água; $10 \mathrm{mg} \mathrm{L}^{-1}$ de CPPU (N-2-cloro-4-piridil)-Nfeniluréia); 1,25 mL L ${ }^{-1}$ de Promalina $\left(\mathrm{GA}_{4+7}+\mathrm{BA}\right) ; 1,25 \mathrm{~mL} \mathrm{~L}^{-1} \mathrm{de}$ Benziladenina (BA) e, $10 \mathrm{mg} \mathrm{L}^{-1}$ de Thidiazuron, aplicados no período entre final da plena floração e a queda de pétalas. Adicionou-se espalhante adesivo siliconado $\operatorname{Silwett}\left(0,5 \mathrm{~mL} \mathrm{~L}^{-1}\right)$ em todos os tratamentos.

Foram utilizadas quatro repetições, utilizando-se de 6 plantas por parcela experimental, sendo avaliadas as duas plantas centrais. $\mathrm{O}$ experimento foi conduzido em um delineamento em parcelas subdivididas, sendo avaliados o efeito dos tratamentos nas parcelas, e dos ciclos vegetativos nas subparcelas. Procedeuse a análise da variância (ANOVA) dos dados, utilizando o pacote estatístico SAS (Statistical Analysis System - versão 8.1).

A primeira aplicação dos fitorreguladores ocorreu em 09 de outubro de 2001, sendo observadas, ao longo do dia, temperatura entre 12,9 a $18,4^{\circ} \mathrm{C}$ e UR do ar entre $61 \%$ e $96 \%$. Em 2002, a aplicação dos fitorreguladores ocorreu em 16 de outubro. Ao longo do referido dia, a temperatura variou de 14,5 a $29,8^{\circ} \mathrm{C}$, e a UR do ar variou de 47 a $96 \%$, iniciando a chover cerca de 4 horas após a aplicação dos tratamentos.

A aplicação dos fitorreguladores foi realizada com pulverizador costal, com pressão média de $40 \mathrm{lbpol}^{-1}$, aspergindose cada planta até o ponto de gotejamento, utilizando um volume de calda de 2.000 Lha $^{-1}$. Para evitar a deriva entre os tratamentos, as plantas utilizadas foram protegidas com lona plástica. Os demais tratamentos fitossanitários foram realizados conforme as Normas Técnicas da PIM (Protas \& Valdebenito-Sanhueza, 2003).

A partir da aplicação dos produtos, foram realizadas coletas quinzenais das maçãs que caíram das plantas, avaliando-se neles a incidência de podridão carpelar. Na colheita, foram coletadas aleatoriamente amostras com 50 frutos por parcela, totalizando 200 frutos por tratamento. As maçãs foram colocadas em redes plásticas, etiquetadas e frigoconservadas a $0,5^{\circ} \mathrm{Ce} 85 \% \mathrm{UR}$, sendo avaliadas após três meses de frigoconservação. Para a avaliação da podridão carpelar, as maçãs foram cortadas no sentido longitudinal, registrando-se o número de maçãs que apresentavam sintomas e/ou sinais da doença. As maçãs que apresentavam sinais foram separadas em classes: maçãs com sinais de fungos nas sementes (Sementes); maçãs com sinais de fungos na cavidade carpelar (Carpelos) e maçãs com podridão na polpa (Podridão).

Para as características de formato, foram medidos o comprimento (C) e o diâmetro (D) de maçãs inteiras com paquímetro, obtendo-se a relação C/D. Posteriormente, as maçãs foram cortadas ao meio, medindo-se o comprimento do tubo calicinal (Figura 3), a abertura calicinal, a distância entre os lóbulos do fruto (Figura 3) e realizando-se a contagem das sementes contidas nos carpelos.

\section{RESULTADOS E DISCUSSÃO}

Durante o período de coleta das maçãs caídas no solo, observaram-se, em ambos os ciclos, que cerca de $50 \%$ dos frutos caídos apresentavam sintomas e/ou sinais da podridão carpelar, em todos os tratamentos. A queda total de maçãs, ocorrida no período da floração até a colheita, variou de 40 a 50 frutos/planta, no ciclo de 2001-2002, e de 110 a 180 frutos/planta no ciclo de 2002-2003 (Figuras 1 e 2). Essa diferença na quantidade de frutos caídos entre os dois anos, pode ser atribuída a diferenças na carga de frutos/planta, uma vez que a frutificação efetiva no ciclo de 2002/2003 foi mais alta. Além disto, em 2001 a plena floração ocorreu no período de 5 a 12 de outubro quando as temperaturas variaram de $14,2^{\circ} \mathrm{C}$ a $15,4^{\circ} \mathrm{C}$. Em 2002 , a plena floração foi mais tardia, ocorrendo na semana de 12 a 19 de outubro, e coincidiu com um período de temperaturas mais altas, variando de $18,3^{\circ} \mathrm{C}$ a $19,9^{\circ} \mathrm{C}$. No primeiro período de coleta de frutos caídos, de 09 de outubro de 2001 a 22 de março de 2002, a precipitação ocorrida foi de $607 \mathrm{~mm}$, bem distribuída ao longo do período, com temperaturas médias variando de $14,1 \mathrm{a} 23{ }^{\circ} \mathrm{C}$, enquanto no segundo período de coleta, de 16 de outubro de 2002 a 26 de março de 2003, a precipitação ocorrida foi de $1.069 \mathrm{~mm}$, com diversos períodos secos seguidos de chuvas intensas, e temperaturas médias variando de 14,9 a $23{ }^{\circ} \mathrm{C}$. Essas são condições propícias a uma formação deficiente do tubo calicinal das maçãs, bem como ao desenvolvimento dos patógenos causadores da podridão carpelar (Spotts, 1990).

Ao final do período de coleta dos frutos, observou-se que não houve diferença significativa na queda de frutos das plantas tratadas com os fitorreguladores no ciclo de 2001-2002, mas foram encontradas diferenças no ciclo de 2002-2003. Neste ciclo, a maior queda de frutos foi observada nos tratamentos com Promalina e Benziladenina, e a maior incidência de podridão carpelar foi observada no tratamento com Benziladenina. Isto pode ser explicado pela ação raleante que estes fitorreguladores provocam nos frutinhos de macieira (Greene, 1993; Leite et al., 2006; Petri et 
al., 2006). A promalina aplicada na floração (estádio F2 até H) tem efeito raleante, mas isto não ocorre quando aplicada após o estádio H (Petri; 2003, Leite et al., 2006). Sabe-se que os raleantes químicos intensificam a queda natural de frutinhos jovens, e diversos trabalhos testando fitorreguladores em macieiras mostram que ocorrem respostas variáveis entre cultivares, doses, épocas de aplicação e idade das plantas. A benziladenina foi indicada como nova alternativa para raleio em macieira, na cultivar Fuji (Petri et al., 2006). No caso da Benziladenina, o efeito raleante tem sido testado com doses entre 50 e $100 \mathrm{mg} \mathrm{L}^{-1}$, e excesso de raleio pode ocorrer em doses acima de $150 \mathrm{mg} \mathrm{L}^{-1}$ (Greene, 1993). Neste experimento, a dose utilizada foi bem mais baixa, pois o principal objetivo era modificar o formato dos frutos, e não provocar o raleio. Apesar disso, deve-se considerar que, no ciclo de 20022003, a frutificação efetiva foi mais alta, e neste caso, a tendência é de que caiam mais frutos/planta, promovendo raleio mesmo em doses mais baixas.

O Thidiazuron e o CPPU foram os tratamentos com a menor queda de frutos e também com a menor incidência de podridão carpelar em frutos jovens (Figuras 1 e 2). Esse efeito confirma dados anteriores, uma vez que o Thidiazuron é recomendado para aumentar a frutificação efetiva. Quando aplicado na cultivar Fuji, em Caçador, aumentou o número de frutos em relação à testemunha (Petri, 2001). O CPPU, testado em maçãs "McIntosh", nas doses de 7,5 e $15 \mathrm{mg} \mathrm{L}^{-1}$, em duas épocas, aumentou o peso dos frutos com baixíssimo raleio (Greene, 1996). O mesmo foi observado por Stern et al. (2003) na cultivar Royal Gala, onde o CPPU, na dose de $10 \mathrm{mg} \mathrm{L}^{-1}$, aumentou o tamanho dos frutos sem causar raleio. Deve-se considerar que a Gala responde melhor aos raleantes que a Fuji; portanto, se esse produto causa baixo efeito raleante em cultivares sensíveis, deverá causar menos ainda em cultivares mais resistentes ao raleio químico.

Após a colheita, ao final do período de frigoconservação, observou-se diferença significativa entre os tratamentos com fitorreguladores e a testemunha nos diferentes níveis da doença (Tabelas 1 e 2). Quando se observa o total de podridão carpelar, não houve diferença significativa entre tratamentos no ano de 2002, mas houve em 2003, sendo que a maior incidência de podridão carpelar ocorreu no tratamento com Benziladenina, diferindo significativamente da testemunha. Esse fitorregulador tem sido apontado como causador de frutos assimétricos, mas isto ocorre apenas em doses acima de $150 \mathrm{mg} \mathrm{L}^{-1}$ (Greene, 1993).

Se observarmos apenas o nível inicial de doença, a maior incidência de infecção de sementes, no ciclo de 2001-2002, sob condições de baixa precipitação pluviométrica, ocorreu nos tratamentos com Thidiazuron e Promalina, os quais diferiram estatisticamente da testemunha (Tabela 1), ou seja, a aplicação de thiadiazuron e promalina proporcionou aumento de sintomas iniciais da podridão carpelar em maçãs em condições climáticas que não são predisponentes à colonização pelos fungos causadores desta doença. Esses dois fitorreguladores também promoveram maior distância entre lóbulos dos frutos, o que predispõe os mesmos à entrada dos fungos causadores da podridão carpelar. Trabalhos anteriores mostram que o TDZ pode causar frutos assimétricos e com deformação na cavidade pistilífera, bem como redução no teor de cálcio e aumento da podridão calicinal (Amarante, 2003; Petri et al., 2001)

No ano seguinte (ciclo de 2002-2003), sob condições normais de precipitação pluviométrica e alta frutificação efetiva, a maior infecção de sementes ocorreu nos tratamentos com CPPU, Benziladenina e Thidiazuron, mas apenas o CPPU diferiu significativamente da testemunha (Tabela 2). Observa-se também, neste ciclo, maior abertura calicinal do que no ano anterior, o que pode ter sido influenciado pelo maior número de frutos/planta $\mathrm{e}$ também pelas condições climáticas, uma vez que não se observaram diferenças significativas entre tratamentos. Normalmente, em anos com cargas maiores, os frutos tendem a ser de menor tamanho e mais achatados. Neste ano, a menor incidência de infecção de sementes foi obtida no tratamento com Promalina, o qual também teve os frutos com o maior comprimento de tubo calicinal, mas não de forma significativa. Isto pode ter reduzido a possibilidade de entrada de fungos via abertura calicinal.

Para as características de formato dos frutos, observouse que a porcentagem de carpelos abertos não variou de forma significativa entre os tratamentos, mas variou entre anos (Tabelas 3; 4 e 5). A relação C/D, a distância entre lóbulos dos frutos e o comprimento do tubo calicinal variaram de forma significativa entre os tratamentos apenas no ciclo de 2001-2002. Neste ciclo, em condições de baixa precipitação pluviométrica, as maçãs tratadas com o CPPU e o Thidiazuron tiveram a menor relação C/ D. O tratamento com thidiazuron também aumentou a distância entre os lóbulos dos frutos de macieira. De acordo com Spotts (1990) e Miller (1959), a abertura dos lóbulos é inversamente relacionada à relação $\mathrm{C} / \mathrm{D}$ dos frutos, e a doença é mais comum em frutos com maior distância entre lóbulos, pois estes apresentam menor relação C/D. O TDZ também teve a maior porcentagem de carpelos abertos, mas não de forma significativa, e promoveu maior infecção de sementes.

Os tratamentos com Promalina e Benziladenina tiveram a mesma relação $\mathrm{C} / \mathrm{D}$ que a testemunha, ou seja, não houve efeito de alongamento dos frutos. Isto concorda com Argenta (1993), que observou que a promalina teve efeito apenas na cultivar Gala, mas não aumentou a relação C/D de frutos de "Fuji". Leite et al. (2006) observaram que a promalina teve efeito de alongamento de frutos na Imperial Gala, mas no caso da "Fuji" os resultados variaram entre anos. Dabul \& Ayub (2006), nas condições do Paraná, não obtiveram efeito de alongamento de frutos com aplicações de promalina na cultivar Gala. Em 2003, a relação C/D, bem como as demais características, não diferiram significativamente entre os tratamentos (Tabela 4).

As características do fruto que podem ter influência sobre a doença variaram entre anos. No ciclo de 2002-2003, a porcentagem de carpelos abertos foi maior, bem como a distância entre os lóbulos do fruto, enquanto a relação C/D e o comprimento do tubo calicinal foram menores (Tabela 5), o que influencia na maior incidência da podridão carpelar. Conforme já discutido anteriormente, a doença é mais comum em frutos com uma razão C/D menor. O aumento dos carpelos abertos em frutos de maçãs "Fuji"/EM-9 está associado com o aumento da distância entre lóbulos e a diminuição do tubo calicinal. 


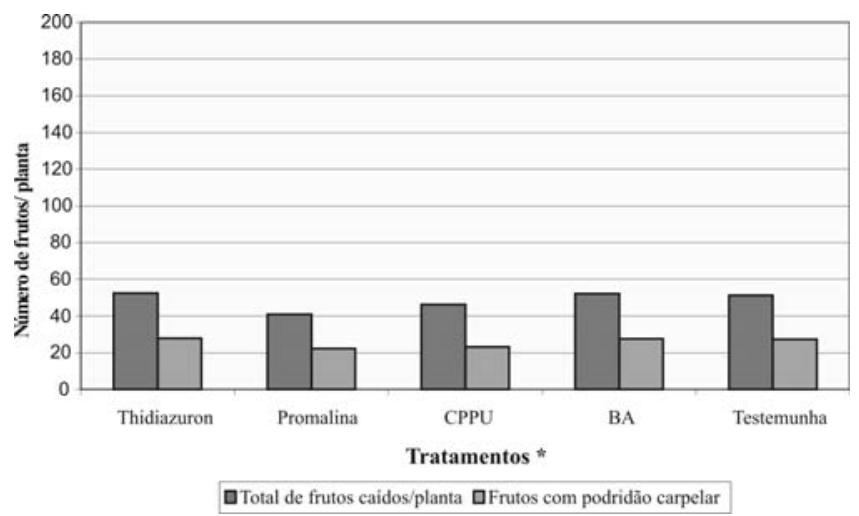

FIGURA 1 - Número total de frutos caídos ao solo, sadios e com podridão carpelar, oriundos de plantas de maçãs "Fuji" tratadas com diferentes fitorreguladores durante a florada do ciclo de 2001-2002. VacariaRS, 2006. *ns pelo teste de Duncan, a 5\%.

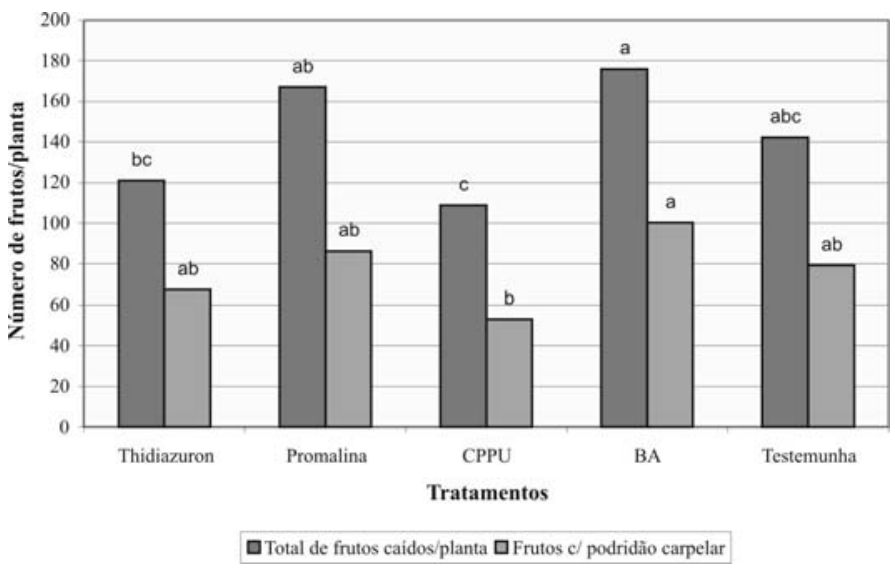

FIGURA 2 - Número total de frutos caídos ao solo, sadios e com podridão carpelar, oriundos de plantas de maçãs "Fuji" tratadas com diferentes fitorreguladores durante a florada do ciclo de 2002-2003. VacariaRS, 2006. * Colunas de mesma cor seguidas pela mesma letra não diferem pelo teste de Duncan, a $5 \%$.

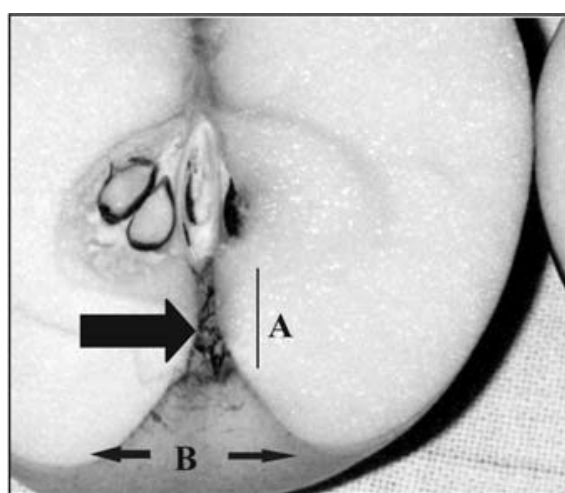

FIGURA 3 - A - Comprimento do tubo calicinal (Distância entre o final dos carpelos e sépalas). B - Distância entre lóbulos.
TABELA 1- Incidência de podridão carpelar em maçãs "Fuji" tratadas com diferentes fitorreguladores na florada e frigoconservadas por três meses (Ciclo de 20012002). Vacaria, 2006

\begin{tabular}{lcccc}
\hline \multicolumn{5}{c}{ Frutos com sintomas ou sinais de podridão carpelar $(\%)^{*}$} \\
Tratamentos & Sementes & Carpelos & Podridão & Total \\
\hline Thidiazuron & $30,0 \mathrm{a}$ & $9,5 \mathrm{~ns}$ & $4,0 \mathrm{~ns}$ & $43,5 \mathrm{~ns}$ \\
Promalina & $23,5 \mathrm{ab}$ & 9,0 & 6,5 & 39,0 \\
BA & $20,0 \mathrm{bc}$ & 12,5 & 5,5 & 38,0 \\
CPPU & $20,0 \mathrm{bc}$ & 12,5 & 5,0 & 37,5 \\
Testemunha & $16,0 \mathrm{c}$ & 11,5 & 6,5 & 34,0 \\
Média & 21,9 & 11,0 & 5,5 & 38,4 \\
\hline
\end{tabular}

* Médias seguidas de mesma letra na coluna não diferem estatisticamente entre si, pelo teste de Duncan, a 5\%. Coeficiente de Variação $=13.35$

TABELA 2 - Incidência de podridão carpelar em maçãs "Fuji" tratadas com diferentes fitorreguladores na florada e frigoconservadas por três meses (Ciclo de 20022003). Vacaria, 2006

\begin{tabular}{lcccl}
\hline \multicolumn{5}{c}{ Frutos com sintomas de podridão carpelar $(\%)^{*}$} \\
Tratamentos & Sementes & Carpelos & Podridão & Total \\
\hline Thidiazuron & $25,5 \mathrm{ab}$ & $10,5 \mathrm{~ns}$ & $6,0 \mathrm{~ns}$ & $42,0 \mathrm{ab}$ \\
Promalina & $16,0 \mathrm{c}$ & 9,5 & 6,0 & $31,5 \mathrm{ab}$ \\
BA & $26,5 \mathrm{ab}$ & 16,0 & 5,0 & $47,5 \mathrm{a}$ \\
CPPU & $27,5 \mathrm{a}$ & 13,0 & 4,5 & $45,0 \mathrm{ab}$ \\
Testemunha & $19,5 \mathrm{bc}$ & 9,0 & 2,0 & $30,5 \mathrm{~b}$ \\
Média & 23,0 & 11,6 & 4,7 & 39,3 \\
\hline
\end{tabular}

* Médias seguidas de mesma letra na coluna não diferem estatisticamente entre si, pelo teste de Duncan, a 5\%. Coeficiente de variação $=15.82$

TABELA 3- Características de maçãs "Fuji" tratadas com diferentes fitorreguladores na florada e frigoconservadas por três meses (Ciclo de 20012002). Vacaria, 2006.

\begin{tabular}{lccccc}
\hline Tratamento & $\begin{array}{c}\text { CA } \\
(\%) * *\end{array}$ & $\begin{array}{c}\text { Relação } \\
\text { C/D }\end{array}$ & $\begin{array}{c}\text { DL } \\
(\mathrm{mm})^{* *}\end{array}$ & $\begin{array}{c}\text { Tubo } \\
(\mathrm{mm}) * *\end{array}$ & $\begin{array}{c}\text { Número } \\
\text { sementes }\end{array}$ \\
\hline Thidiazuron & $38,0 \mathrm{a}$ & $0,86 \mathrm{~b}$ & $26,6 \mathrm{a}$ & $8,12 \mathrm{ab}$ & $6,10 \mathrm{c}$ \\
Promalina & $35,0 \mathrm{a}$ & $0,88 \mathrm{a}$ & $26,1 \mathrm{ab}$ & $7,67 \mathrm{~b}$ & $6,25 \mathrm{bc}$ \\
BA & $35,0 \mathrm{a}$ & $0,89 \mathrm{a}$ & $25,6 \mathrm{ab}$ & $8,02 \mathrm{ab}$ & $5,90 \mathrm{c}$ \\
CPPU & $37,0 \mathrm{a}$ & $0,85 \mathrm{~b}$ & $26,1 \mathrm{ab}$ & $8,35 \mathrm{a}$ & $7,07 \mathrm{a}$ \\
Testemunha & $30,0 \mathrm{a}$ & $0,88 \mathrm{a}$ & $25,0 \mathrm{~b}$ & $7,92 \mathrm{ab}$ & $6,65 \mathrm{ab}$ \\
\hline
\end{tabular}

* Médias seguidas da mesma letra na coluna não diferem estatisticamente entre si, pelo teste de DMS, a 5\%.

** $\mathrm{CA}=$ carpelos abertos; $\mathrm{DL}=$ distância entre lóbulos do fruto; $\quad$ Tubo = tubo calicinal. 
TABELA 4 - Características de maçãs "Fuji” tratadas com diferentes fitorreguladores na florada e frigoconservadas por três meses (Ciclo de 20022003). Vacaria, 2006.

\begin{tabular}{lccccc}
\hline Tratamento & $\begin{array}{c}\text { CA } \\
(\%)^{* *}\end{array}$ & $\begin{array}{c}\text { Relação } \\
\text { C/D }\end{array}$ & $\begin{array}{c}\text { DL } \\
(\mathrm{mm})^{* *}\end{array}$ & $\begin{array}{c}\text { Tubo } \\
(\mathrm{mm})^{* *}\end{array}$ & $\begin{array}{c}\text { Número } \\
\text { sementes }\end{array}$ \\
\hline Thidiazuron & $49,5 \mathrm{~ns} *$ & $0,82 \mathrm{~ns}$ & $26,7 \mathrm{~ns}$ & $5,77 \mathrm{~ns}$ & $6,80 \mathrm{~ns}$ \\
Promalina & 41,0 & 0,82 & 26,3 & 6,30 & 6,47 \\
BA & 50,5 & 0,81 & 26,9 & 6,02 & 6,70 \\
CPPU & 48,0 & 0,81 & 27,2 & 6,05 & 6,55 \\
Testemunha & 38,0 & 0,81 & 26,2 & 6,02 & 6,65 \\
\hline
\end{tabular}

* Médias na coluna não diferem estatisticamente, pelo teste de DMS, a 5\%. ** $\mathrm{CA}=$ carpelos abertos; $\mathrm{DL}=$ distância entre lóbulos do fruto; $\quad$ Tubo = tubo calicinal

TABELA 5 - Variação das características de maçãs "Fuji" tratadas com diferentes fitorreguladores na florada e frigoconservadas por três meses. Vacaria, 2006.

\begin{tabular}{cccccc}
\hline Ano & $\begin{array}{c}\text { CA } \\
(\%)^{* *}\end{array}$ & $\begin{array}{c}\text { Relação } \\
\text { C/D }\end{array}$ & $\begin{array}{c}\text { DL } \\
(\mathrm{mm})^{* *}\end{array}$ & $\begin{array}{c}\text { Tubo } \\
(\mathrm{mm})^{* *}\end{array}$ & $\begin{array}{c}\text { Número } \\
\text { Sementes }\end{array}$ \\
\hline 2002 & $35,0 \mathrm{~b}$ & $0,87 \mathrm{a}$ & $25,9 \mathrm{~b}$ & $8,02 \mathrm{a}$ & $6,39 \mathrm{a}$ \\
2003 & $45,4 \mathrm{a}$ & $0,81 \mathrm{~b}$ & $26,7 \mathrm{a}$ & $6,03 \mathrm{~b}$ & $6,63 \mathrm{a}$ \\
\hline \multicolumn{2}{l}{ Médias }
\end{tabular}

(etra na coluna não diferem estatisticamente entre si, pelo teste de DMS, a $5 \%$.

** $\mathrm{CA}=$ carpelos abertos; $\mathrm{DL}=$ distância entre lóbulos do fruto; $\quad$ Tubo = tubo calicinal;

\section{CONCLUSÕES}

1-O thiadiazuron e a promalina, aplicados no final da plena floração de macieiras cv. Fuji, proporcionam aumento dos sintomas iniciais de podridão carpelar em frutos maduros.

2-A benziladenina aplicada no final da plena floração de macieiras cv Fuji aumenta a queda de frutos jovens e a incidência de podridão carpelar em frutos maduros.

3-O thidiazuron, aplicado no final da plena floração em macieiras cv. Fuji, reduz a relação C/D e aumenta a distância entre lóbulos de frutos.

\section{REFERÊNCIAS}

AMARANTE, C.V.T.; MEGGUER, C. A; BLUM, L.E.B. Effect of preharvest spraying with thidiazuron on fruit quality and maturity of apples. Revista Brasileira de Fruticultura, Jaboticabal, v. 25, n.1, p.59-62, 2003.

ARGENTA, L.C.; PETRI, J.L. ; MONDARDO, M. Efeito de piridiluréias e $\mathrm{GA}_{4+7}+\mathrm{BA}$ sobre o crescimento de maçãs cvs Gala e Fuji. Revista Brasileira de Fruticultura, Jaboticabal, v. 15, n.1, p. 111-116, 1993.

BANGERTH, F.; SCHRODER, M. Strong synergistic effects of gibberelins with the synthetic cytokinin N-(2-chloro-4-pyridyl)$\mathrm{N}$-phenylurea on parthenocarpic fruti set and some other fruit characteristics of apple. Plant Growth Regulation, Dordrecht, v. 15, n.3, p.293-302, 1994

BONETI,J.I.; KATSURAYAMA,Y. Podridão Carpelar da Macieira. In: REUNIÃO ANUAL DE FITOSSANIDADENACULTURADA MACIEIRA, 1998/99, São Joaquim, SC. Relatório... São Joaquim, SC: Epagri. Estação Experimental de São Joaquim, 1999. 40 p

CURRY,E.A.; GREENE,D.W. CPPU influences fruit quality, fruit set, return bloom, and preharvest drop of apples. Hortscience, Alexandria, v. 28, n.2,p. $115-119,1993$

CZERMAINSKI,A B.C.; VALDEBENITO-SANHUEZA,R.M.; MELLO,G.W.; FREIRE, J. M. Podridão carpelar das maçãs: estimativas de perdas no período 1999 a 2002 em Vacaria,RS. Bento Gonçalves: Embrapa CNPUV, 2002. (Boletim Técnico, 42).

DABUL, A.N.G.; AYUB, R.A. Efeito da promalina (6BA+ GA4+7) no crescimento e desenvolvimento de frutos de macieira (Malus domestica, Borkh.) cv. Gala. Semina:Ciências Agrárias, Londrina, v.27, n.2, p.199-204, 2006.

ELLIS, M.A Colonization of Delicious apple fruits by Alternaria spp and effect of fungicide sprays on moldy-core. Plant Disease, St. Paul, v. 67, n. 2, p. 150-152, 1983.

GREENE, D.W. A Review of the use of Benzyladenine (BA) as a Chemical Thinner for apples. Acta Horticulturae, The Hague, v. 329, p.231-236, 1993.

GREENE, D.W. Influence of CPPU on fruit quality and storage potential of "McIntosh" apples. Journal of Tree Fruit Production, Binghamton, v. 1, p. 87-97, 1996.

LEITE, G.B.; PETRI, J.L.; BASSO, C. Promalin effect on "Imperial Gala" and "Fuji" apple trees fructification. Acta Horticulturae, The Hague, v. 727, p.269-278, 2006.

MILLER, P.M. Open calyx tubes as a factor contributing to carpel discoloration and decay of apples. Phytopathology, St. Paul, v. 49, p. 520-523, 1959 .

PETRI, J.L.; SCHUCK, E.; LEITE, G.B. Efeito do thidiazuron(TDZ) na frutificação de fruteiras de clima temperado. Revista Brasileira de Fruticultura, Jaboticabal, v.23, n.3, 2001.

PETRI,J.L. Formação de flores, polinização e fertilização. In: EPAGRI. Manual da cultura da macieira. Florianópolis: EPAGRI, 2002. p. 229-259.

PETRI, J.L. Efeito do uso da Promalina na cultura da macieira. In: ENCONTRO NACIONAL SOBRE FRUTICULTURADE CLIMA TEMPERADO, 6., 2003, Fraiburgo. Anais... Fraiburgo: Epagri, 2003, v.1, p. 197-202. 
PETRI, J.L.; LEITE, G.B.; BASSO,C. Chemical thinning of "Fuji" apples growing in a mild winter climate. Acta Horticulturae, The Hague, v. 727, p.429-436, 2006

PROTAS, J. F. S.; VALDEBENITO-SANHUEZA, R. M. Produção integrada de frutas: o caso da maçã no Brasil. Bento Gonçalves: EMBRAPA Uva e Vinho, 2003. 192 p.

REUVENI, M. SHEGLOV, D. SHEGLOV, N.; BEN-ARIE, R. PRUSKY, D. Sensitivity of Red Delicious apple fruit at various phonologic stages to infection by Alternaria alternata and moldy core control. European Journal of Plant Pathology, Dordrecht, v.108, n.5, p. $421-427,2002$.
REUVENI, M. SHEGLOV, D.; COHEN, Y. Control of moldy-core decay in apple fruits by â-aminobutyric acids and potassium phosphites. Plant Disease, St. Paul, v.87, p.933 - 936, 2003.

SAS INSTITUTE. System for Information. Versão 8.1. Cary 2000. SPOTTS, R.A Moldy Core and Core Rot. In: JONES, A L.; ALDWINCKLE,H.S. Compendium of apple and pear disease. St Paul: APS Press, 1990. p. 29-30.

STERN, R.A.;BEN-ARIE,R.; NERIA, O.; FLAISHMAN, M..CPPU and BA increased fruit size of "Royal Gala" (Malus domestica) apple in a warm climate. The Journal of Horticultural Science and Biotechnology, Valência, v.78, n.3, p.297-302, 2003. 